\title{
Temperature dependence of surface roughening during homoepitaxial growth on $\mathrm{Cu}(001)$
}

\author{
C. E. Botez and P. F. Miceli \\ Department of Physics and Astronomy, University of Missouri-Columbia, Columbia, Missouri 65211 \\ P. W. Stephens \\ Department of Physics, State University of New York, Stony Brook, New York 11794
}

(Received 20 March 2001; published 11 September 2001)

\begin{abstract}
X-ray scattering has been used to study the roughening of the $\mathrm{Cu}(001)$ surface during homoepitaxial growth, as a function of temperature. Between 370 and $160 \mathrm{~K}$, the mean-square roughness $\sigma^{2}$, obtained from specular reflectivity data, was found to increase as a power law $\sigma^{2}=\Theta^{2 \beta}$ for coverages $\Theta$, ranging from 3 to 96 ML. The roughening exponent $\beta$ was observed to depend on the temperature of the substrate: it monotonically increases with decreasing temperature from $\beta \approx \frac{1}{3}$ at $T=370 \mathrm{~K}$ to $\beta \approx \frac{1}{2}$, at $T=200 \mathrm{~K}$. At $110 \mathrm{~K}$ a smoother growth re-enters in the presence of a large vacancy concentration in the deposited film.
\end{abstract}

DOI: $10.1103 /$ PhysRevB.64.125427

PACS number(s): $61.10 . \mathrm{Kw}, 68.55 . \mathrm{Jk}$

\section{INTRODUCTION}

In spite of being driven by the same basic processes, such as impingement, diffusion, and incorporation of the adatoms into the surface, epitaxial growth can progress in very different modes, which leads to a large variety of surface morphologies. Recently, much attention has been attracted by growth processes where the evolving surface morphology exhibits regular pyramidlike structures with a well-defined lateral separation and selected mound slope. This form of three-dimensional growth, which has been observed for the $\operatorname{Ag}(001),{ }^{1,2} \mathrm{Fe}(001),{ }^{3} \mathrm{Cu}(001),{ }^{4} \mathrm{Ag}(111),{ }^{5}$ and $\mathrm{Pt}(111)$ (Ref. 6) homoepitaxy, originates from instabilities caused by an additional energy barrier that opposes the transport of the diffusing atoms downward over the step edges [EhrlichSchwoebel (ES) effect]. ${ }^{7}$ These surfaces are not self-affine (because they show a distinct lateral length scale) and, therefore, the dynamic scaling approach ${ }^{8}$ is not suitable for their description. Yet, the evolution with coverage of their meansquare roughness $\sigma^{2}$, as predicted by models that incorporate the ES effect, ${ }^{9-11}$ exhibits the same simple power law as the one proposed by the dynamic scaling theories:

$$
\sigma^{2}=\Theta^{2 \beta},
$$

where $\Theta$ is the coverage and $\beta$ is a growth exponent. Several experiments, using either direct imaging ${ }^{1-3,12}$ or diffraction techniques, ${ }^{4,5}$ confirmed the behavior described by Eq. (1) and also showed that the growth exponent $\beta$ is not universal, but depends on the material and on the temperature of the substrate. However, the temperature dependence of growth on these surfaces is not yet fully explored, and many of its particular aspects, such as the microscopic mechanisms that drive the reentrant smooth growth observed on $\mathrm{Pt}(111),{ }^{13}$ $\mathrm{Cu}(001),{ }^{4}$ and $\mathrm{Ag}(001),{ }^{1,2}$ are still a subject of ongoing discussion. ${ }^{1,14}$ This is partly due to the very limited number of experiments that systematically address the temperature dependence of roughening.

In particular, the temperature dependence of $\beta$ is expected to contain information on the kinetic details of growth as well as on the magnitude of the ES barrier ${ }^{10}$ and, therefore, its experimental determination and comparison with theoretical predictions presents a special interest. Theoretically, several scenarios are possible. If no smoothening mechanism, other than diffusion, is present, the growth becomes progressively rougher (i.e., $\beta$ increases) with decreasing $T$, since the lowering of the temperature reduces the barrier-crossing probability by decreasing the diffusion rate of the monomers. Below a specific temperature, a sufficiently large Schwoebel barrier becomes insurmountable, leading to "Poisson growth" with $\beta \approx \frac{1}{2} .{ }^{10,15}$ Experimentally, this behavior was observed for the $\operatorname{Ag}(111)$ homoepitaxy. ${ }^{5}$ On the other hand, if an additional smoothening mechanism, such as "downward funneling" 16 is present, the growth in the low-temperature range may become smoother as the temperature is lowered and a different " $\beta$ vs $T$ " behavior is expected: ${ }^{10}$ at high enough temperatures, where the downward funneling is inoperative, $\beta$ increases with decreasing $T$ until it reaches a maximum, which may be one half or smaller (depending on the strength of the step barrier) and it eventually decreases in the low-temperature range, where the increasing step density progressively enhances the downward funneling for lower $T$. Such a reentrant smooth growth behavior has been suggested for $\mathrm{Cu}(001)$ homoepitaxy in a recent helium-atom beam scattering study, ${ }^{4}$ which found a considerably smoother growth at $T=160 \mathrm{~K}\left(\beta \approx \frac{1}{4}\right)$ than at $T=200 \mathrm{~K}\left(\beta \approx \frac{1}{2}\right)$. Surprisingly, however, a previous experiment employing the same technique did not indicate a similar reentrant behavior at $T$ $=160 \mathrm{~K} \cdot{ }^{17}$ There, the amplitude of the out-of-phase intensity oscillations was observed to steadily decrease with coverage as $T$ was lowered from 300 to $150 \mathrm{~K}$, showing that the growth becomes progressively rougher within this temperature interval. A slight recovery of the oscillation amplitude, suggesting a smoother growth, was only observed at $T$ $=100 \mathrm{~K}$.

Motivated by this discrepancy and in an attempt at gaining more insight into the temperature dependence of roughening on (001) surfaces, we have used x-ray diffraction to investigate the homoepitaxial growth on $\mathrm{Cu}(001)$ within a broad temperature range of 370-100 K. Traditional x-ray reflectivity methods, enhanced by the use of synchrotron radiation, are particularly accurate for surface-roughness mea- 
surements and are also very sensitive to defects below the surface that induce a lattice mismatch between the deposited film and the underlying bulk crystal. ${ }^{18,19}$ Our specular reflectivity data show that the mean-square roughness of the growing film is well described by the power law of Eq. (1), for coverages ranging from 3 to $96 \mathrm{ML}$, and for all temperatures between 370 and $160 \mathrm{~K}$. We find that the corresponding roughening exponent $\beta$ amounts to $\approx \frac{1}{2}$ at low temperatures $(160$ and $200 \mathrm{~K})$, and steadily decreases above $200 \mathrm{~K}$, reaching $\beta \approx \frac{1}{3}$ at $T=370 \mathrm{~K}$. We do not observe the reentrant smooth growth at $T=160 \mathrm{~K}$, reported in Ref. 4. Instead, we find that the smoother growth reenters at lower temperatures in the presence of a large vacancy concentration $(\sim 2 \%)$ in the deposited film. This result is consistent with the partial recovery of the out-of-phase intensity oscillations at $T$ $=100 \mathrm{~K}$ that was observed in Ref. 17 .

\section{EXPERIMENTAL PROCEDURE}

The measurements were carried out on the SUNY X3B2 beamline at the National Synchrotron Light Source (NSLS), using a customized ultrahigh vacuum (UHV) $\mathrm{x}$-ray diffractometer. A double crystal sagitally focused monochromator ${ }^{20}$ was utilized to select radiation of wavelength $0.842 \AA$. The surface of the 12 -mm-diameter $\times 2$-mm-thick $\mathrm{Cu}$ sample was oriented perpendicular to the [001] direction to within $0.1^{\circ}$ by mechanical polishing and subsequently annealed $(900 \mathrm{~K})$ for several days in UHV (base pressure $<10^{-10}$ Torr) in order to remove the damage from polishing. The sample was then cooled to $300 \mathrm{~K}$ and sputtered for $1 \mathrm{~h}$ with $\mathrm{Ar}^{+}$at $p$ $=10^{-5}$ Torr (the acceleration voltage was $1 \mathrm{KV}$ and the sputtering current $10 \mu \mathrm{A} / \mathrm{cm}^{2}$ ). Further cycles of 1 -h annealing at $850 \mathrm{~K}$ and $15-\mathrm{min} \mathrm{Ar}^{+}$sputtering were used to remove the impurities detected by Auger-electron spectroscopy (AES). As shown by $\mathrm{x}$-ray-scattering data, this procedure allowed us to obtain clean surfaces that are virtually flat at the atomic level (rms roughness $<0.5 \AA$ ), have a mosaic spread of less than $0.005^{\circ}$, and consist of facets whose average size (inplane correlation length) is $5000 \AA$. Cu was evaporated from a resistively heated crucible mounted in a Knudsen cell. One sputter/anneal cycle was performed prior to each growth series. The deposition rate (at the sample position) was calibrated by using a quartz monitor and, more precisely by following the intensity oscillations of the antiphase specular beam-a rate of $1 \mathrm{ML} / \mathrm{min}$ was chosen. Liquid-nitrogen cooling and resistive heating were simultaneously used to stabilize $( \pm 1 \mathrm{~K})$ the temperature of the substrate during deposition. Before each deposition series, the temperature of the sample was accurately determined by direct measurement of the lattice constant and the cleanliness of the surface was verified by AES. Once a given coverage was attained the deposition was stopped to allow the x-ray-scattering measurements to be performed. Data were collected by scanning across the specular rod (transverse scans) over an extended range of values of the surface-normal scattering wave vector $Q_{z}$, at temperatures between 370 and $110 \mathrm{~K}$. At each $Q_{z}$, the specular reflectivity was obtained from the corresponding transverse profile by subtracting the diffuse scattering component. ${ }^{18}$

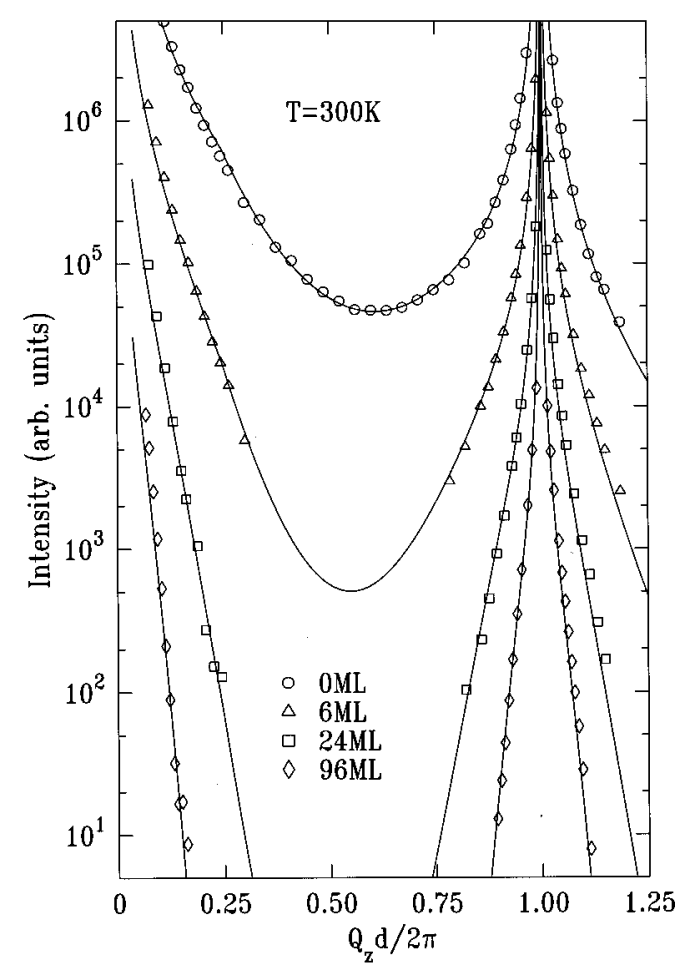

FIG. 1. Specular reflectivity measured for the clean (starting) $\mathrm{Cu}(001)$ surface (circles) and for rough surfaces with $6 \mathrm{ML}$ (triangles), $24 \mathrm{ML}$ (squares), and $96 \mathrm{ML}$ (diamonds), deposited at $T$ $=300 \mathrm{~K}$. The curves are vertically shifted for clarity. The reflectivity dampens with the increasing coverage as the surface becomes progressively rougher. The solid lines represent best fits of Eq. (2), which allow the determination of $\sigma^{2}$.

\section{RESULTS AND DISCUSSION}

Figure 1 shows the specular reflectivity data (open symbols), measured at $T=300 \mathrm{~K}$, for a smooth starting $\mathrm{Cu}(001)$ surface (circles) and for rough surfaces with coverages of 6 ML (triangles), $24 \mathrm{ML}$ (squares), and $96 \mathrm{ML}$ (diamonds). We observe that the reflectivity progressively dampens with coverage as the surface becomes increasingly rougher. As expected, ${ }^{18}$ this effect is more pronounced for $Q_{z}$ values far from the in-phase position. The solid lines are best fits to the data of a real-space model where the rough surface is assumed to exhibit height fluctuations described by discrete Gaussian statistics. In the kinematic limit, the specular reflectivity from such a surface is given by the expression ${ }^{21}$

$$
\begin{gathered}
R\left(Q_{z}\right)=c P F\left(Q_{z}\right)\left|f\left(Q_{z}\right)\right|^{2} \times \exp \left[-\frac{1}{2}\left\langle u^{2}\right\rangle Q_{z}^{2}\right] \\
\times \frac{\exp \left[-4 \frac{\sigma^{2}}{d^{2}} \sin ^{2}\left(\frac{Q_{z} d}{2}\right)\right]}{Q_{z}^{2} \sin ^{2}\left(\frac{Q_{z} d}{2}\right)} .
\end{gathered}
$$

Here, $c$ is a scaling constant (since the reflectivity was not measured in absolute units), $P$ is the polarization factor, $F\left(Q_{z}\right)$ includes the geometrical correction for the number of $\mathrm{x}$ rays striking the surface, and $f\left(Q_{z}\right)$ is the atomic form 


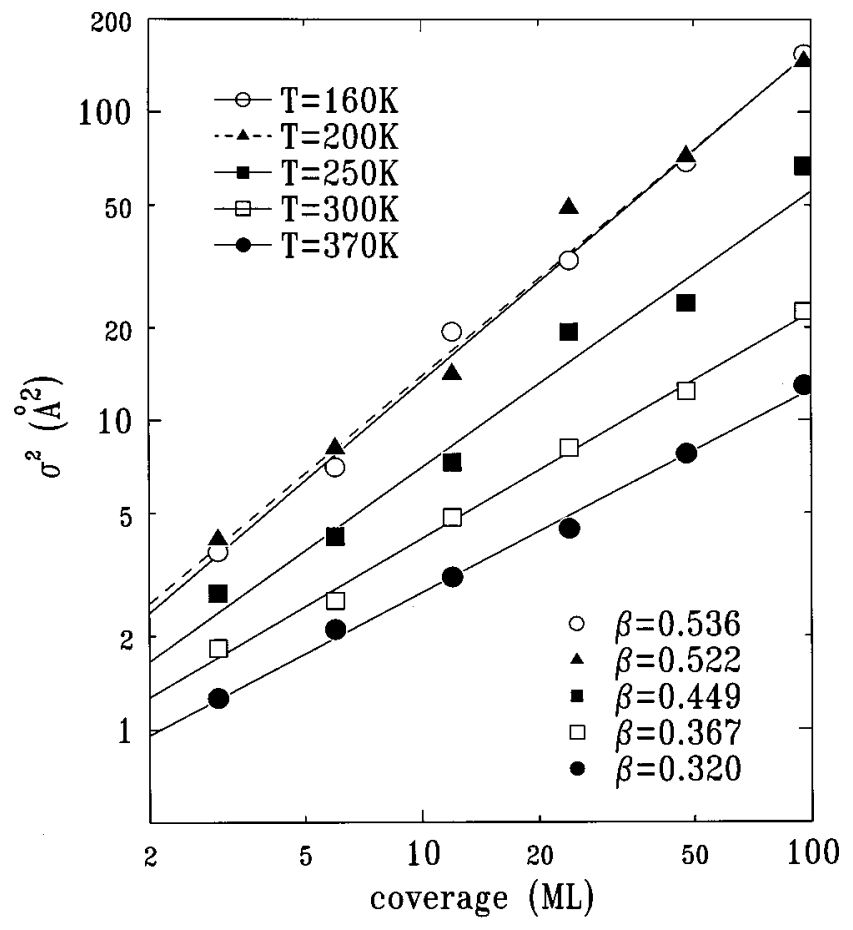

FIG. 2. Mean-square roughness of the $\mathrm{Cu}(001)$ surface $\sigma^{2}$ as a function of coverage $\Theta$ at five different temperatures. The lines are least-square fits of $\sigma^{2}=\Theta^{2 \beta}$. Both the mean-square roughness (measured at a given coverage) and the exponent $\beta$ decrease with the increasing temperature of the substrate.

factor. $\left\langle u^{2}\right\rangle$ denotes the mean-square amplitude of the surface-normal atomic vibrations and $d$ is the separation between the atomic planes along the surface normal. $\sigma^{2}$ is the mean-square roughness (surface width), defined by $\sigma^{2}$ $=\sum_{i} N^{-1}\left(h_{i}-\bar{h}\right)^{2}$, where $\bar{h}$ represents the average height of the surface, $N$ is the number of surface lattice sites, and $h_{i}$ is the height at site $i$. In our analysis, the mean-square amplitudes of the surface-normal atomic vibrations were set to their temperature-dependent values, calculated according to the Debye model, ${ }^{22}$ leaving the rms roughness $\sigma$ as the only variable parameter, since the scaling constant $c$ was fixed by the clean starting surface. As shown in Fig. 1, this procedure gives excellent fits within a broad coverage range $(3 \leqslant \Theta$ $\leqslant 96 \mathrm{ML}$ ), allowing a precise determination of $\sigma^{2}$.

The coverage dependence of the mean-square roughness, obtained from fits of Eq. (2) to the reflectivity data, is shown in Fig. 2 for different substrate temperatures between 370 and $160 \mathrm{~K}$. We observe that, at all temperatures, the roughness evolution is very well described by the power law $\sigma^{2}$ $=\Theta^{2 \beta}$. The resulting roughening exponent $\beta$ is temperature dependent: as shown in Fig. $3, \beta \approx \frac{1}{2}$ at low temperatures $(160$ and $200 \mathrm{~K})$ and it monotonically decreases with the increasing temperature, reaching $\approx \frac{1}{3}$ at $T=370 \mathrm{~K}$.

We now discuss the observed temperature dependence of $\beta$. It is generally believed that $\beta \approx \frac{1}{2}$ characterizes the growth when the interlayer mass transport is completely inhibited by a perfectly reflecting step barrier. ${ }^{10,15}$ Monte Carlo simulations ${ }^{10,11}$ have shown that two temperature-dependent mechanisms can lead to (and enhance the rate of) barrier

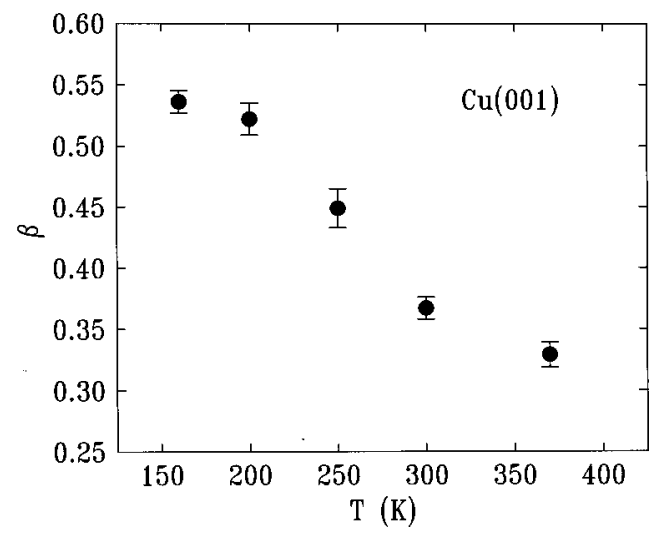

FIG. 3. Temperature dependence of the roughening exponent $\beta$, for the homoepitaxial growth on $\mathrm{Cu}(001)$. At low temperatures $(160$ and $200 \mathrm{~K}$ ), $\beta \approx \frac{1}{2}$; above $200 \mathrm{~K}, \beta$ steadily decreases with increasing temperature, reaching $\approx \frac{1}{3}$ at $T=370 \mathrm{~K}$.

crossing: thermal activation, where the mobility of the adatoms progressively increases with increasing $T$, and some high-order-crossing effects such as "downward funneling" 16 or "transient mobility," 23 which are significant only at low temperatures. Thus, for temperatures small enough to inhibit the thermal activation over the step edges, but large enough to make the high-order step-crossing processes inoperative (if these are present at all for that given surface), a sufficiently large step barrier can become perfectly reflecting, leading to $\beta \approx \frac{1}{2}$. This is what we observe for $\mathrm{Cu}(001)$ at $T$ $=160$ and $200 \mathrm{~K}$. Above $200 \mathrm{~K}$, the thermal activation progressively grows with the increasing temperature and we observe that $\beta$ smoothly decreases, reaching $\approx \frac{1}{3}$ at $T=370 \mathrm{~K}$. A previous study of $\operatorname{Ag}(111)$ homoepitaxy $y^{5}$ showed a qualitatively similar behavior, but $\beta \approx \frac{1}{2}$ was found to persist over a broader temperature range, $150-300 \mathrm{~K}$, and a much steeper decrease of $\beta$ was observed above $300 \mathrm{~K}$. It is therefore very likely that the temperature-dependent roughening of the two surfaces, $\mathrm{Cu}(001)$ and $\mathrm{Ag}(111)$, is dominated by the same kinetic processes, but the specific values of certain parameters (step-barrier magnitude, diffusion rates, etc.) lead to quantitatively different " $\beta$ vs $T$ " behaviors.

At $200 \mathrm{~K}$, our results for both the mean-square roughness (measured at a given coverage) and for the exponent $\beta$ are in excellent (quantitative) agreement with the findings of a Heatom scattering study. ${ }^{4}$ At $160 \mathrm{~K}$, however, our data show no evidence of the reentrant smooth growth reported there, which consists of a dramatic reduction of $\sigma^{2}$ (measured in the range of tens of ML's of coverage) associated with a drop of $\beta$ to $50 \%$ of its $200-\mathrm{K}$ value. Instead, we found that the coverage dependence of $\sigma^{2}$ and the corresponding exponent $\beta$, at $T=160 \mathrm{~K}$, are very similar to those observed at $200 \mathrm{~K}$, as can be seen in Fig. 2. On the other hand, our results are consistent with those of a previous He-atom scattering study, ${ }^{17}$ where the amplitude of the out-of-phase intensity oscillations was found to steadily decrease with $T$ in the interval between 300 and $150 \mathrm{~K}$, showing a progressively rougher growth with decreasing temperature and no reentrant behavior at $160 \mathrm{~K}$. A slight recovery of the oscillation amplitude, possibly indicating a reentrant smoother growth, was 


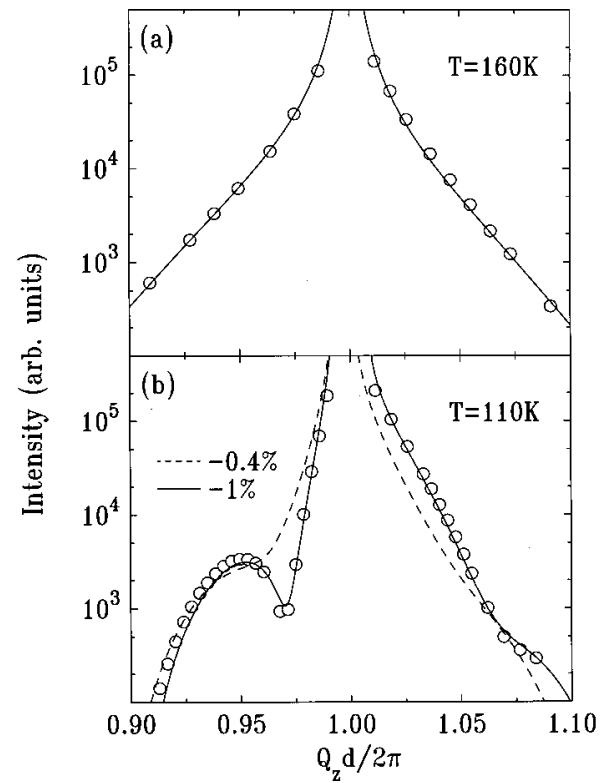

FIG. 4. Specular reflectivity from the $\mathrm{Cu}(001)$ surface, with 15 ML deposited at (a) $T=160 \mathrm{~K}$ and (b) $T=110 \mathrm{~K}$ (open symbols). At $160 \mathrm{~K}$, the data is well described by Eq. (2) (solid line) while, at $110 \mathrm{~K}$ a real-space model that includes a large compressive strain in the deposited film is necessary to fit the interference fringes and the pronounced asymmetry of the reflectivity. The best fit (solid line) yields a compressive strain of $-1 \%$. The dashed line, which corresponds to a strain of a smaller amplitude $(-0.4 \%)$, significantly deviates from the data.

only observed at $T=100 \mathrm{~K}$. Our low-temperature data, described below, are in agreement with this observation.

Figure 4 shows the effect of reducing the temperature from 160 to $110 \mathrm{~K}$ on the reflectivity from $\mathrm{Cu}(001)$ with 15 ML deposited. At $160 \mathrm{~K}$, the reflectivity measured around the (002) Bragg reflection (open circles) is very well described by Eq. (2) [solid line in Fig. 4(a)]. On the other hand, at 110 $\mathrm{K}$, the reflectivity line shape exhibits thin-film interference fringes as well as pronounced asymmetry about the (002) Bragg reflection [Fig. 4(b)]. We find that both of these features arise from the presence of a compressive strain in the deposited film. Indeed, the data at $110 \mathrm{~K}$ are excellently described by a model where, in addition to considering the surface roughness, we allow the surface-normal interlayer spacings in the film $d_{\text {film }}$ to differ from their value in the bulk crystal $d_{\text {bulk }}$. A best fit to this model [solid line in Fig. 4(b)] yields $\left(d_{\text {film }}-d_{\text {bulk }}\right) / d_{\text {bulk }}=-1 \%$. The sensitivity of the measured reflectivity to the magnitude of the strain is appreciable: as shown by the dashed line in Fig. 4(b), a strain of a smaller magnitude $(-0.4 \%)$ would lead to a reflectivity curve that significantly deviates from the data. In regards to the origin of the compressive strain observed here, we emphasize that an accidental low-temperature surface contamination cannot explain this result, since impurities would cause a lattice expansion rather than the observed contraction. Moreover, an extremely large impurity concentration would be necessary to account for the magnitude of the observed strain, while Auger spectroscopy shows clean surfaces at all temperatures. Alternatively, if we consider the possibil-

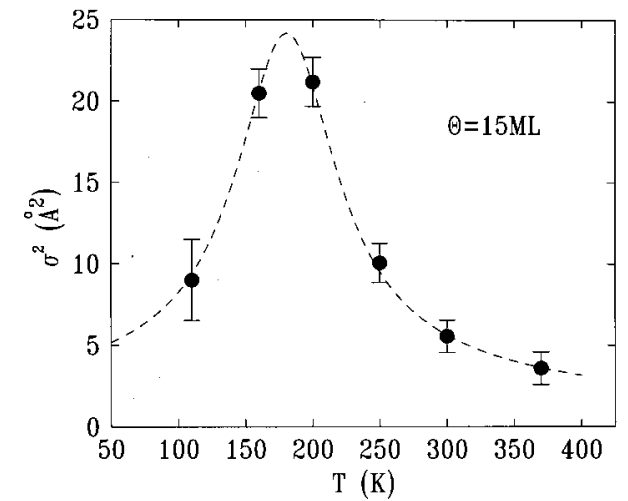

FIG. 5. Temperature dependence of the mean-square roughness $\sigma^{2}$ measured at a constant coverage $\Theta=15 \mathrm{ML}$ (solid symbols); the dashed line is a guide to the eye. Initially, $\sigma^{2}$ increases with decreasing $T$, as the growth becomes rougher. At $110 \mathrm{~K}$, however, smoother growth reenters $\left(\sigma^{2}\right.$ decreases as $T$ is lowered) in the presence of a large vacancy concentration.

ity of stacking faults on (111) facets, we expect only a small strain leading to an expanded lattice. Consequently, we believe that the compressive strain is induced by a large vacancy concentration, which is likely to appear in the deposited film when the growth occurs at very low temperatures. By using the linear relationship between the concentration of point defects and the strain in a film ${ }^{24}$ we estimate that a $2 \%$ vacancy concentration is present in the $\mathrm{Cu}$ film deposited at $T=110 \mathrm{~K}$. A comprehensive analysis of the vacancy formation during the low-temperature roughening of $\mathrm{Cu}(001)$ will be presented elsewhere. ${ }^{25}$ For our present study, the important quantity resulting from the best fit to the $110-\mathrm{K}$ reflectivity data is the surface mean-square roughness $\sigma^{2}=9 \AA^{2}$, which is less than half of its value at $T=160 \mathrm{~K}$. Thus, a smoother growth reenters at $T=110 \mathrm{~K}$. This behavior is demonstrated in Fig. 5, where the temperature dependence of the mean-square roughness of $\mathrm{Cu}(001)$ with $15 \mathrm{ML}$ deposited is presented. We observe that as the film is grown at progressively lower temperatures, $\sigma^{2}$ initially increases by almost one order of magnitude in the interval between 370 and $200 \mathrm{~K}$, but decreases significantly by $T=110 \mathrm{~K}$, where the growth appears to occur in the presence of a large vacancy concentration.

A similar reentrant smooth growth behavior was very recently found in a scanning tunneling microscopy (STM) study of $\operatorname{Ag}(001)$ homoepitaxy, ${ }^{1}$ where it was observed that the rms surface roughness $(\sigma)$ of 25 -ML-thick $\mathrm{Ag} / \mathrm{Ag}(001)$ films increases when $T$ is reduced from 300 to $200 \mathrm{~K}$, but then decreases when the temperature is further lowered from 200 to $130 \mathrm{~K}$. Except for a slight shift in temperature [for $\mathrm{Ag}(001)$ the smooth growth reenters at a higher $T]$ this behavior bears a remarkable resemblance to our " $\sigma^{2}$ vs $T$ " data, shown in Fig. 5. Thus, it appears that the reentrant smooth growth is indeed a characteristic of the homoepitaxy on (001) surfaces, as predicted by previous theoretical studies. ${ }^{10,16}$ These theories attribute the reentrant behavior to the downward funneling of the atoms deposited at step edges to lower fourfold hollow absorption sites. They also predict that the downward funneling is continuously enhanced by 
decreasing $T$, so that the growth on (001) surfaces becomes increasingly smoother when $T$ approaches $0 \mathrm{~K}^{16}$ Interestingly, this latter prediction is not confirmed in the STM data in Ref. 1, where an increasingly rougher growth was observed with the decreasing $T$ from 120 to $50 \mathrm{~K}$. To explain this novel "reentrant rough growth," the authors of Ref. 1 suppose that at very low temperatures the depositing atoms might get caught on the sides of the larger microprotrusions (which are more numerous at low $T$ ), which is a process that would partially inhibit the downward funneling and lead to a rougher growth. This "restricted downward funneling" is believed to lead to the formation of overhangs and internal voids, as also predicted by molecular dynamics simulations for low-temperature growth. ${ }^{26}$

The present $\mathrm{Cu} / \mathrm{Cu}(001)$ experiment as well as our previous observations for $\mathrm{Ag}$ homoepitax $\mathrm{y}^{25}$ indicate the presence of vacancies for the low- $T$ growth on (001) surfaces, but, unlike what has been proposed ${ }^{1}$ for $\operatorname{Ag}(001)$, we observe a reentrant smooth growth on $\mathrm{Cu}(001)$ in the presence of a large $(2 \%)$ vacancy concentration. This suggests that, whatever the mechanism, the formation of vacancies during the low- $T \mathrm{Cu}(001)$ homoepitaxy is unrelated to the microprotrusions (which yield rougher surfaces) found in the abovementioned simulations. Here, we speculate that vacancies might arise from the slow terrace and edge diffusion that is also known to be responsible for the dendritically shaped mounds found ${ }^{1,27}$ in low-temperature metal homoepitaxy. With restricted diffusion, there could be sites that do not fill and, even with a reduced ES barrier, the timescale for filling the vacancy from a higher-level terrace would be quite slow. In this picture, it is not clear whether the reentrant smooth growth is caused by the vacancies or by a changing surface morphology. For example, an effectively reduced ES barrier associated with the long dendritic "fingers" that develop at low temperature has been suggested as an explanation for the reentrant smooth growth on $\operatorname{Pt}(111) \cdot{ }^{13}$ Alternatively, it is possible that the strain associated with the vacancies could change the ES barrier itself. In any case, the presence of vacancies seems to be associated with substantial changes in the " $\sigma$ vs $T$ " behavior, suggesting the existence of a close relationship between the vacancy formation and the kinetic mechanisms that govern the low-temperature homoepitaxial growth on (001) surfaces.

In summary, we have used x-ray scattering to study the temperature dependence of roughening during the homoepitaxial growth on $\mathrm{Cu}(001)$. At temperatures between 370 and $160 \mathrm{~K}$, we found that the mean-square roughness evolves with the coverage as a power law: $\sigma^{2}=\Theta^{2 \beta}$. The roughening exponent increases with the decreasing temperature from $\beta \approx \frac{1}{3}$ at $T=370 \mathrm{~K}$ to $\beta \approx \frac{1}{2}$ at $T=200 \mathrm{~K}$ but, contrary to previous observations, ${ }^{4}$ remains unchanged when the temperature is lowered to $160 \mathrm{~K}$. Between 370 and $200 \mathrm{~K}$ we find that, for a given coverage, $\sigma^{2}$ becomes progressively larger at lower substrate temperatures. At $T=110 \mathrm{~K}$, however, reentrant smooth growth is observed concomitantly with a considerable strain in the growing film, produced by a large vacancy concentration.

\section{ACKNOWLEDGMENTS}

Support is acknowledged from the National Science Foundation under contracts (P.W.S.) DMR-9202528 and (P.F.M. and C.E.B.) DMR-9623827 and the Midwest Superconductivity Consortium (MISCON) under DOE Grant No. DH-FG02-90ER45427. The SUNY X3 beamline was supported by the DOE, under Contract No. DE-FG0286ER45231, and the NSLS was supported by the DOE, Division of Material Sciences, and Division of Chemical Sciences. We thank Ian Robinson for the $\mathrm{Cu}$ crystal and for valuable discussions.
${ }^{1}$ K. J. Caspersen, C. R. Stoldt, A. R. Layson, M. C. Bartelt, P. A. Thiel, and J. W. Evans, Phys. Rev. B 63, 085401 (2001).

${ }^{2}$ G. Constantini, F. Buatier de Mongeot, C. Boragno, and U. Valbusa, Surf. Sci. Lett. 459, L487 (2000).

${ }^{3}$ J. A. Stroscio, D. T. Pierce, M. Stiles, A. Zangwill, and L. M. Sander, Phys. Rev. Lett. 75, 4246 (1995).

${ }^{4}$ H.-J. Ernst, F. Fabre, R. Folkerts, and J. Lapujoulade, Phys. Rev. Lett. 72, 112 (1994).

${ }^{5}$ W. C. Elliott, P. F. Miceli, T. Tse, and P. W. Stephens, Phys. Rev. B 54, 17938 (1996); W. C. Elliott, P. F. Miceli, T. Tse, and P. W. Stephens, in Surface Diffusion: Atomistic and Collective Processes, Vol. 360 of NATO Advanced Studies Institute Series B-Physics, edited by M. C. Tringides (Plenum Press, New York, 1997), pp. 209.

${ }^{6}$ M. Bott, T. Michely, and G. Comsa, Surf. Sci. 272, 161 (1992).

${ }^{7}$ J. Villain, J. Phys. I 1, 19 (1991); G. Ehrlich and F. G. Hudda, J. Chem. Phys. 44, 1039 (1966); R. L. Schwoebel and E. J. Shipsey, J. Appl. Phys. 37, 3682 (1966).

${ }^{8}$ For a review, see Solids Far From Equilibrium: Growth Morphology and Defects, edited by C. Godriche (Cambridge University,
New York, 1991), pp. 432 and 479; F. Family, Physica A 168, 561 (1990).

${ }^{9}$ M. Siegert and M. Plischke, Phys. Rev. Lett. 73, 1517 (1994).

${ }^{10}$ M. C. Bartelt and J. W. Evans, Phys. Rev. Lett. 75, 4250 (1995).

${ }^{11}$ J. G. Amar and F. Family, Phys. Rev. B 54, 14742 (1996).

${ }^{12}$ J.-K. Zou and J. F. Wendelken, Phys. Rev. Lett. 78, 2791 (1997); R. A. Lukaszew, Y. Sheng, C. Uher, and R. Clarke, Appl. Phys. Lett. 76, 724 (2000).

${ }^{13}$ R. Kunkel, B. Poelsema, L. K. Verheij, and G. Comsa, Phys. Rev. Lett. 65, 733 (1990).

${ }^{14}$ P. Smilauer, M. R. Wilby, and D. D. Vvedensky, Phys. Rev. B 47, 4119 (1993).

${ }^{15}$ Z. Zhang, J. Detch, and H. Metiu, Phys. Rev. B 48, 4972 (1993).

${ }^{16}$ J. W. Evans, D. E. Sanders, P. A. Thiel, and A. E. DePristo, Phys. Rev. B 41, 5410 (1990).

${ }^{17}$ H.-J. Ernst, F. Fabre, and J. Lapujoulade, Surf. Sci. Lett. 275, L682 (1992).

${ }^{18}$ I. K. Robinson and D. J. Tweet, Rep. Prog. Phys. 55, 559 (1992).

${ }^{19}$ I. K. Robinson, Phys. Rev. B 33, 3830 (1986).

${ }^{20}$ P. W. Stephens, P. J. Eng, and T. Tse, Rev. Sci. Instrum. 64, 374 (1993). 
${ }^{21}$ W. C. Elliott, P. F. Miceli, T. Tse, and P. W. Stephens, Physica B 221, 65 (1996).

${ }^{22}$ B. D. Cullity, Elements of X-Ray Diffraction (Addison-Wesley, Reading, 1956).

${ }^{23}$ W. F. Egelhoff and I. Jacob, Phys. Rev. Lett. 62, 921 (1989).

${ }^{24}$ L. D. Landau and E. M. Lifshitz, Theory of Elasticity (Pergamon, New York, 1970).
${ }^{25}$ C. E. Botez, W. C. Elliott, P. F. Miceli, and P. W. Stephens (unpublished).

${ }^{26}$ C. L. Kelchner and A. E. DePristo, Surf. Sci. 393, 72 (1997).

${ }^{27}$ M. Hohage, M. Bott, M. Morgenstern, Z. Zhang, T. Michely, and G. Comsa, Phys. Rev. Lett. 76, 2366 (1996); M. Kalff, G. Comsa, and T. Michely, ibid. 81, 1255 (1998). 